\title{
Antitumor agents 279. Structure-activity relationship and in vivo studies of novel 2-(furan-2-yl)naphthalen-1-ol (FNO) analogs as potent and selective anti-breast cancer agents
}

\author{
Yizhou Donga ${ }^{\mathrm{a}}$, Kyoko Nakagawa-Goto ${ }^{\mathrm{a}}$, Chin-Yu Lai ${ }^{\mathrm{a}}$, Yoon Kim ${ }^{\mathrm{C}}$, Susan L. Morris-

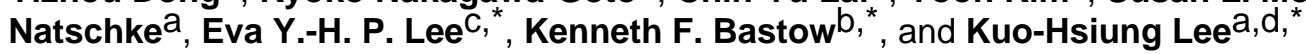 \\ aNatural Products Research Laboratories, UNC Eshelman School of Pharmacy, University of \\ North Carolina, Chapel Hill, North Carolina 27599-7568 \\ ${ }^{b}$ Division of Medicinal Chemistry and Natural Products, UNC Eshelman School of Pharmacy, \\ University of North Carolina, Chapel Hill, North Carolina 27599-7568 \\ 'Department of Biological Chemistry and Department of Developmental \& Cell Biology, University \\ of California, Irvine, CA 92697-4037 \\ ${ }^{d}$ Chinese Medicine Research and Development Center, China Medical University and Hospital, \\ Taichung, Taiwan
}

\begin{abstract}
In our ongoing modification study of neo-tanshinlactone (1), we discovered 2-(furan-2yl)naphthalen-1-ol (FNO) derivatives $\mathbf{3}$ and $\mathbf{4}$ as a new class of anti-tumor agents. To explore structure-activity relationships (SAR) of this scaffold, 18 new analogs, 6-12 and 14-24, were designed and synthesized. The $\mathrm{C} 11$-esters $\mathbf{7}$ and $\mathbf{1 2}$ displayed broad anti-tumor activity $\left(\mathrm{ED}_{50} 1.1-\right.$ $4.3 \mu \mathrm{g} / \mathrm{mL}$ against seven cancer cell lines), while C11-hydroxymethyl $\mathbf{1 4}$ showed unique selectivity against the SKBR-3 breast cancer cell line ( $\left.\mathrm{ED}_{50} 0.73 \mu \mathrm{g} / \mathrm{mL}\right)$. Compounds $\mathbf{1 5}$ and 22 displayed potent and selective anti-breast tumor activity $\left(\mathrm{ED}_{50} 1.7\right.$ and $0.85 \mu \mathrm{g} / \mathrm{mL}$, respectively, against MDA-MB-231). The SAR results demonstrated that the substitutions from the ring-opened lactone ring $\mathrm{C}$ of $\mathbf{1}$ are critical to the anti-tumor potency as well as the apparent tumor-tissue type selectivity. Treatment with $\mathbf{3}$ in $\mathrm{Brcal}^{f 11 / f 11} p 53^{f 5 \& 6 / f 5 \& 6} \mathrm{Cr} e^{c}$ mice models significantly inhibited the proliferation of mammary epithelial cells and branching of mammary glands.
\end{abstract}

\section{Keywords \\ 2-(furan-2-yl)naphthalen-1-ol analogs; structure-activity relationships; anti-breast tumor agents}

Neo-tanshinlactone (1), a natural product from Salvia miltiorrhiza, and its analog, 4-ethyl neo-tanshinlactone (2), are potent and selective anti-breast cancer agents (Figure 1). ${ }^{1-3}$ In our prior studies, they exhibited high tumor tissue-type as well as breast cancer cell line selectivity. The selective anti-breast tumor activity of $\mathbf{2}$ in mice models is consistent with the

\footnotetext{
(C) 2010 Elsevier Ltd. All rights reserved.

*Corresponding authors. (K.F.B) Tel: 919-966-7633, Fax: 919-966-0204, ken_bastow@unc.edu. (E.Y.H.P.L) Tel: 949-824-9766, elee@uci.edu. (K.H.L) Tel: 919-962-0066; Fax: 919-966-3893; khlee@unc.edu.

Publisher's Disclaimer: This is a PDF file of an unedited manuscript that has been accepted for publication. As a service to our customers we are providing this early version of the manuscript. The manuscript will undergo copyediting, typesetting, and review of the resulting proof before it is published in its final citable form. Please note that during the production process errors may be discovered which could affect the content, and all legal disclaimers that apply to the journal pertain.
} 
results in vitro. ${ }^{3}$ In our continuing study, we explored how the individual rings A-D in the molecule influence the in vitro anti-breast tumor activity. The results led to the discovery of a novel class of anti-breast cancer agents, 2-(furan-2-yl)naphthalen-1-ol (FNO) analogs (e.g., 3 and 4) by opening ring-C. ${ }^{4}$ Our previous studies also explored the preliminary SAR and proved that the $\mathrm{C} 8$ and $\mathrm{C} 11$ substituents can greatly affect both potency and selectivity. FNO analog 3 showed significant potency $\left(\mathrm{ED}_{50} 0.3 \mu \mathrm{g} / \mathrm{mL}\right.$ ) and selectivity against the ZR-7-51 (ER+, HER2+) cell line compared with other cancer cell lines tested, while 4 exhibited activity against all cancer cell lines tested. ${ }^{4} \mathrm{We}$ wanted to use these promising results to design novel analogs with better pharmaceutical profiles and develop them as clinical trials candidates.

The initial studies showed that $\mathrm{Et}, \mathrm{H}$, and $\mathrm{Me}$ groups are preferred at the $\mathrm{C} 4, \mathrm{C} 14$, and $\mathrm{C} 15$ positions, respectively, of the FNO skeleton, and we retained this substitution pattern in our current study. ${ }^{4}$ However, we expanded the identities of the groups at the C8 and C11 positions ( $\mathbf{3}$ and $\mathbf{4}$ contain hydroxyl/carboxylic acid and methyl ether/methyl ester, respectively). Some of the different combinations at these positions included ether/ carboxylic acid, ether/ester, ether/amide, and ether/substituted methyl. We incorporated groups with different sizes and electrostatic effects to establish SAR and develop new lead compounds.

As shown in Scheme 1, carboxylic acids $\mathbf{5}$ and $\mathbf{6}$, synthesized by the method reported before, ${ }^{4}$ were converted to esters $\mathbf{7 , 8}$, and $\mathbf{1 2}$, respectively, with thionyl chloride and the appropriate alcohols at room temperature..$^{5}$ In addition, treatment of $\mathbf{5}$ with Lawesson's reagent led to methylthioate 9,3 with methanamine to amide $\mathbf{1 0}$, and with hydroxybenzotriazole (HOBt) to benzotriazole ester 11. Meanwhile, known diol 13 was treated with iodomethane or iodoethane in the presence of $\mathrm{Cs}_{2} \mathrm{CO}_{3}$ to generate phenoxyethers 14 and 15, respectively. ${ }^{5}$ The primary hydroxyl groups of 14 and 15 were alkylated with iodomethane, iodoethane, or 1,1,1-trifluoro-2-iodoethane in the presence of sodium hydride to obtain 17, 18, 20, and 23. Acetates 16, 21, and 24 were synthesized by acetylation of 13-15 with $\mathrm{Ac}_{2} \mathrm{O}$ in pyridine. Benzylic bromination of 14 with triphenylphosphine, bromine, and imidazole afforded bromomethyl 19. Ester 22 was obtained by reaction of $\mathbf{1 4}$ with 1-ethyl-3-(3-dimethylaminopropyl) carbodiimide (EDCI), 4dimethylaminopyridine (DMAP), and 3-(diethylamino)propanoic acid hydrochloride.

All newly synthesized analogs 6-12 and 14-246 were tested for cytotoxic activity against a focused panel of human tumor cell lines according to previously published methods (Table 1) ${ }^{3}$ Cell lines included A549 (non small cell lung cancer), DU145 (prostate cancer cell line), KB (nasopharyngeal carcinoma) and KB-VIN (MDR KB subline selected using vincristine), MDA-MB-231 (estrogen receptor negative basal-like breast cancer), SK-BR-3 (estrogen receptor negative, HER2 over-expressing luminal-like breast cancer), and ZR-75-1 (estrogen receptor positive breast cancer, HER2 over-expressing luminal-like breast cancer).

Among analogs (6-12) with ether $\left(\mathrm{OR}^{1}\right)$ and ester or similar $\left(\mathrm{R}^{2}\right)$ groups at $\mathrm{C} 8$ and $\mathrm{C} 11$, respectively, compound 12 (ethyl ether, methyl ester) showed comparable activity to 4 (methyl ether, methyl ester). The potencies were affected greatly by the $\mathrm{R}^{2}$ functional group, with the following rank order: COOMe (12) COOEt (7) $>\operatorname{CSOMe~(9)~} \gg \operatorname{COOPr}(\mathbf{8})$, $\mathrm{CONHMe} \mathrm{(10),} \mathrm{COOH}(\mathbf{6})$. Free carboxylic acid, larger ester, thioester, and amide groups $(6,8,9,10)$ dramatically reduced or eliminated potency. The results also suggested that the combination of ether and carboxylic ester at $\mathrm{C} 8$ and $\mathrm{C} 11$, respectively, may lead to broad inhibition of cancer cell lines from different tissues $(\mathbf{4 , 7 , 9}$, and 12). Small ester groups, including COOMe $(\mathbf{4}, \mathbf{1 2})$ and COOEt $(\mathbf{7})$, were preferred at $\mathrm{C} 11$. 
Among analogs (14-24) with ether $\left(\mathrm{OR}^{3}\right)$ and substituted methyl $\left(\mathrm{CH}_{2} \mathrm{R}^{4}\right)$ groups at $\mathrm{C} 8$ and $\mathrm{C} 11$, respectively, compound $\mathbf{1 4}$ with a $\mathrm{C} 11$ hydroxymethyl group $\left(\mathrm{R}^{4}=\mathrm{OH}\right)$ displayed significant potency and unique selectivity, being more than nine-fold more active against the SKBR-3 breast cancer cell line (ED ${ }_{50} 0.73 \mu \mathrm{g} / \mathrm{mL}$ ) compared with other cancer cell lines tested $\left(\mathrm{ED}_{50} 6.6->10 \mu \mathrm{g} / \mathrm{mL}\right)$. Interestingly, compound $\mathbf{1 5}$, in which the $\mathrm{C} 8$ methyl ether $\left(\mathrm{OR}^{3}=\mathrm{OMe}\right)$ of $\mathbf{1 4}$ is replaced by an ethyl ether $\left(\mathrm{OR}^{3}=\mathrm{OEt}\right)$, showed high potency and selectivity against ZR-75-1 and MDA-MB-231 (ED ${ }_{50} 1.4$ and $1.7 \mu \mathrm{g} / \mathrm{mL}$, respectively). These results indicate that the size of the $\mathrm{R}_{3}$ group could change the apparent cell line selectivity. Compounds 15 and $22\left(\mathrm{R}_{4}=\mathrm{OCOCH}_{2} \mathrm{CH}_{2} \mathrm{NEt}_{2} ; \mathrm{ED}_{50} 1.7 \mu \mathrm{g} / \mathrm{mL}, \mathrm{ZR}-75-1\right.$; $0.85 \mu \mathrm{g} / \mathrm{mL}$, MDA-MB-231) displayed comparable activity against all cell lines tested. Changing the $\mathrm{R}^{4}$ group in the $\mathrm{C} 11$ substituent to other groups (e.g., acetate, bromide, methoxy) generally led to lower potency. Although diacetate $16\left(\mathrm{R}^{4}=\mathrm{OR}^{3}=\mathrm{OAc}\right)$ was completely inactive, monoacetates 21 and $\mathbf{2 4}\left(\mathrm{R}^{4}=\mathrm{OAc}, \mathrm{R}_{2}=\mathrm{Me}\right.$ and Et, respectively) were active against ZR-75-1 (21 and 24) and MDA-MB-231 (24). The bromomethyl compound $\mathbf{1 9}\left(\mathrm{R}^{4}=\mathrm{Br}\right)$ was also selective against $\mathrm{ZR}-75-1$, and $\mathbf{2 3}\left(\mathrm{R}^{4}=\mathrm{OMe}\right)$ showed moderate activity against MDA-MB-231. These results suggested that the identity of the $\mathrm{R}^{4}$ group might affect selectivity against ZR-75-1. Interestingly, $\mathbf{2 3}$ and $\mathbf{2 4}$ showed some growth inhibition against the P-glycoprotein over-expressing KB-VIN cell line, but no inhibition against the parent KB cell line. Further work will be needed to determine whether the apparent MDR-selectivity of $\mathbf{2 3}$ and $\mathbf{2 4}$ is significant.

Subsequently, we evaluated the anti-proliferation activity of 3. BRCAl is the first cloned familial breast cancer susceptibility gene that is expressed in all cells. ${ }^{7,8} B R C A 1$ mutations significantly increase breast and ovarian cancer risk in female carriers. Male $B R C A l$ carriers also have increased breast cancer risk, but the risk factor is lower than that of $B R C A 2$ carriers. ${ }^{8}$ The BRCA1 protein plays a critical role in DNA damage repair, cell cycle checkpoint control, and transcriptional regulation. ${ }^{9,10} \mathrm{Brcal}^{f 11 / f 11} p 53^{f 5 \& 6 / f 5 \& 6} \mathrm{Cr} e^{c}$ mice show extensive mammary epithelial proliferation that can be effectively inhibited by the progesterone antagonist mifepristone (RU 486). ${ }^{11}$ Thus, this mouse model can provide critical information on in vivo activity of novel experimental anti-breast tumor agents.

To assess the effects of $\mathbf{3}$ on mammary epithelial proliferation in vivo, we treated Brcal ${ }^{f 11 / f 11} p 53^{f 5 \& 6 / f 5 \& 6} \mathrm{Cr} e^{c}$ mice and wild-type mice with 3 . $^{12-14}$ As previously reported, mammary glands from control vehicle-treated Brcal ${ }^{f 11 / f 11} p 53^{f 5 \& 6 / 55 \& 6} \mathrm{Cre} e^{c}$ mice showed remarkable accumulation of side branches and extensive alveolar formation compared with similarly treated wild-type mice at three months of age (Figure 2A, a \& c). Compared with vehicle-treated control, mammary gland branching points in $\mathrm{Brcal} \mathrm{f}^{f 1 / f 11} p 53^{f 5 \& 6 / f 5 \& 6} \mathrm{Cre} e^{c}$ and wild-type mice were significantly reduced, with a $70 \pm \%$ reduction in the former and a $29 \pm \%$ reduction in the latter (Figure 2A, b \& c; Figure 2B).

To address the inhibitory effects of $\mathbf{3}$ on mammary epithelial cell proliferation, bromodeoxyuridine (BrdU) was used for labeling the S-phase. Drinking water containing BrdU $(1 \mathrm{mg} / \mathrm{mL})$ was supplied to mice during the last three days of treatment. BrdU-positive mammary epithelial cells were quantified and found to be about 3.5 fold higher in the vehicle-treated Brcal/p53-deficient mice than wild-type mice (Figure 3A, a \& c; Figure 3B), indicating that the proliferation of mammary gland in Brcal/p53-deficient mice is 3.5 fold higher than wild-type. In 3-treated wild-type mice, BrdU positive mammary epithelial cells were undetectable, while BrdU-positive cells were found occasionally in the extracellular matrix of the mammary gland (Figure 3A, b). These findings indicated that the inhibitory effects of $\mathbf{3}$ are likely specific to the mammary epithelial cells. Treatment of Brcal/p53deficient mice with $\mathbf{3}$ dramatically reduced BrdU-positive mammary epithelial cells (83\%) (Figure 3A, d; Figure 3B). This result implied that 3 inhibited the onset of the cell-cycle Sphase in both wild-type and Brcal/p53-deficient mammary epithelial cells. 
In summary, we established the SAR of FNO analogs and developed lead compounds with high potency and selectivity. The results indicated that the $\mathrm{C} 8$ and $\mathrm{C} 11$ substituents, which are analogous to the opened ring- $\mathrm{C}$, play a critical role in both potency and apparent tumortissue selectivity. Compounds $\mathbf{7}$ and $\mathbf{1 2}$ displayed broad activity spectrum against all cancer cell lines tested, while $\mathbf{1 5}$ and $\mathbf{2 2}$ showed more than four-fold greater potency against ZR-751 and MDA-MB-231 (ED 501.7 and $0.85 \mu \mathrm{g} / \mathrm{mL}$, respectively, against ZR-751), and 14 showed more than nine-fold greater potency against SKBR-3 (ED $50.73 \mu \mathrm{g} / \mathrm{mL})$. Based on this study, SAR results are as follows. (1) The combination of ether at C8 and ester at C11 may lead to broad inhibition of cancer cell lines from different tissues, and the combination of ether at C8 and carboxylic acid at C11 is not favored. (2) Small ester groups at C11 are preferred. (3) An acetoxymethyl or bromomethyl group at C11 might induce selectivity against ZR-75-1.

Proliferation of mammary epithelial cells is regulated by ovarian hormones. ${ }^{15}$ Several studies have indicated that $B R C A l$ suppresses the hyperactivation of estrogen and progesterone receptors. ${ }^{16}$ Mutation in BRCAl leads to expansion of the mammary epithelial cells. ${ }^{17}$ The robust expansion of mammary epithelial cells in the Brcal mouse model of human breast cancer provides increased sensitivity and accuracy for identification and evaluation of compounds with clinically significant anti-proliferation activity in vivo. Although molecular targets of $\mathbf{3}$ are currently unknown, in vivo studies, including the branching of mammary glands and the prevention of mammary epithelial cells entering Sphase, showed that $\mathbf{3}$ has significant effects on preventing mammary epithelial cell proliferation in both wild-type and Brcal/p53-deficient mice.

\section{Acknowledgments}

This work was supported by NIH grant CA-17625 from the National Cancer Institute, awarded to K.H. Lee.

\section{References and notes}

1. Wang X, Bastow KF, Sun CM, Lin YL, Yu HJ, Don MJ, Wu TS, Nakamura S, Lee KH. J Med. Chem 2004;47:5816. [PubMed: 15509181]

2. Wang X, Nakagawa-Goto K, Bastow KF, Don MJ, Lin YL, Wu TS, Lee KH. J. Med. Chem 2006;49:5631. [PubMed: 16942038]

3. Dong Y, Shi Q, Pai HC, Peng CY, Pan SL, Teng CM, Nakagawa-Goto K, Yu D, Liu YN, Wu PC, Bastow KF, Morris-Natschke SL, Brossi A, Lang JY, Hsu JL, Hung MC, Lee EY, Lee KH. J. Med. Chem 2010;53:2299. [PubMed: 20148565]

4. Dong Y, Shi Q, Liu Y-N, Wang X, Bastow KF, Lee K-H. J. Med. Chem 2009;52:3586. [PubMed: 19425534]

5. Dong Y, Shi Q, Nakagawa-Goto K, Wu PC, Bastow KF, Morris-Natschke SL, Lee KH. Bioorg. Med. Chem. Lett 2009;19:6289. [PubMed: 19819136]

6. Materials and Methods. ${ }^{1} \mathrm{H}$ NMR spectra were measured on a 300 or $400 \mathrm{MHz}$ Varian Gemini 2000 spectrometer using TMS as internal standard. The solvent used was $\mathrm{CDCl}_{3}$ unless indicated. Mass spectra were measured on a Shimadzu LC-MS2010 instrument. Thin-layer chromatography (TLC) and preparative TLC were performed on precoated silica gel GF plates purchased from Merck, Inc. Biotage Flash+ or Isco Companion systems were used for flash chromatography. Silica gel (200-400 mesh) from Aldrich, Inc., was used for column chromatography. All other chemicals were obtained from Aldrich, Inc, and Fisher, Inc. Analogs 6-12 and 14-24 were prepared by the optimized methods described in our previous paper. Key compound 14 was $>95 \%$ pure on the basis of HPLC conditions. Analytical data of target compounds are shown as follows. Compound 6: ${ }^{1} \mathrm{H}$ NMR (300 MHz, CD $\left.{ }_{3} \mathrm{COCD}_{3}, \mathrm{ppm}\right): \delta 8.10(\mathrm{~d}, 1 \mathrm{H}, J=7.5 \mathrm{~Hz}, \mathrm{Ar}-H), 7.89(\mathrm{~d}, 1 \mathrm{H}, J=8.7 \mathrm{~Hz}, \mathrm{Ar}-$ $H$ ), $7.57(\mathrm{~m}, 2 \mathrm{H}, \mathrm{Ar}-H), 7.46(\mathrm{~m}, 2 \mathrm{H}, \mathrm{Ar}-H \& \mathrm{OCH}), 3.82\left(\mathrm{q}, 2 \mathrm{H}, J=6.9 \mathrm{~Hz}, \mathrm{OCH}_{2} \mathrm{CH}_{3}\right), 3.13(\mathrm{q}$, $\left.2 \mathrm{H}, J=7.5 \mathrm{~Hz}, \mathrm{CH}_{2} \mathrm{CH}_{3}\right), 2.25\left(\mathrm{~d}, 3 \mathrm{H}, J=1.2 \mathrm{~Hz}, \mathrm{CH}_{3}\right), 1.36\left(\mathrm{t}, 3 \mathrm{H}, J=7.2 \mathrm{~Hz}, \mathrm{CH}_{2} \mathrm{CH}_{3}\right), 1.25$ (t, $\left.3 \mathrm{H}, J=7.2 \mathrm{~Hz}, \mathrm{OCH}_{2} \mathrm{CH}_{3}\right)$. MS m/z $323\left(\mathrm{M}^{+}-1\right)$; Compound 7: ${ }^{1} \mathrm{H} \mathrm{NMR}\left(300 \mathrm{MHz}, \mathrm{CDCl}_{3}, \mathrm{ppm}\right)$ : 
$\delta 8.10(\mathrm{~d}, 1 \mathrm{H}, J=8.1 \mathrm{~Hz}, \mathrm{Ar}-H), 7.84(\mathrm{~d}, 1 \mathrm{H}, J=8.4 \mathrm{~Hz}, \mathrm{Ar}-H), 7.55(\mathrm{~d}, 1 \mathrm{H}, J=9.0 \mathrm{~Hz}, \mathrm{Ar}-H)$, $7.46(\mathrm{t}, J 1 \mathrm{H},=8.4 \mathrm{~Hz}, \mathrm{Ar}-H), 7.39(\mathrm{~d}, 1 \mathrm{H}, J=8.7 \mathrm{~Hz}, \mathrm{Ar}-H), 7.35(\mathrm{~d}, 1 \mathrm{H}, J=0.9 \mathrm{~Hz}, \mathrm{OCH}), 4.17$ (q, $\left.2 \mathrm{H}, J=7.2 \mathrm{~Hz}, \mathrm{OCH}_{2} \mathrm{CH}_{3}\right), 3.70\left(\mathrm{~s}, 3 \mathrm{H}, \mathrm{OCH}_{3}\right), 3.12\left(\mathrm{q}, 2 \mathrm{H}, J=7.5 \mathrm{~Hz}, \mathrm{CH}_{2} \mathrm{CH}_{3}\right), 2.26$ (d, $\left.3 \mathrm{H}, J=1.2 \mathrm{~Hz}, \mathrm{CH}_{3}\right), 1.39$ (t, $\left.3 \mathrm{H}, J=7.5 \mathrm{~Hz}, \mathrm{CH}_{2} \mathrm{CH}_{3}\right), 1.07$ (t, $\left.3 \mathrm{H}, J=7.2 \mathrm{~Hz}, \mathrm{OCH}_{2} \mathrm{CH}_{3}\right) . \mathrm{MS}$ $m / z 339\left(\mathrm{M}^{+}+1\right)$; Compound 8: ${ }^{1} \mathrm{H}$ NMR $\left(400 \mathrm{MHz}, \mathrm{CDCl}_{3}, \mathrm{ppm}\right): \delta 8.09$ (d, $1 \mathrm{H}, J=8.0 \mathrm{~Hz}, \mathrm{Ar}-$ $H), 7.83(\mathrm{~d}, 1 \mathrm{H}, J=8.8 \mathrm{~Hz}, \operatorname{Ar}-H), 7.53(\mathrm{~d}, 1 \mathrm{H}, J=8.8 \mathrm{~Hz}, \operatorname{Ar}-H), 7.45(\mathrm{t}, 1 \mathrm{H}, J=8.8 \mathrm{~Hz}, \mathrm{Ar}-H)$, $7.38(\mathrm{~d}, 1 \mathrm{H}, J=6.4 \mathrm{~Hz}, \mathrm{Ar}-H), 7.34(\mathrm{~d}, 1 \mathrm{H}, J=1.2 \mathrm{~Hz}, \mathrm{OCH}), 4.07$ (t, $2 \mathrm{H}, J=6.8 \mathrm{~Hz}, \mathrm{OCH}_{2}$ $\mathrm{CH}_{2} \mathrm{CH}_{3}$ ), 3.70 (s, $3 \mathrm{H}, \mathrm{OCH}_{3}$ ), 3.11 (q, $\left.2 \mathrm{H}, J=7.6 \mathrm{~Hz}, \mathrm{CH}_{2} \mathrm{CH}_{3}\right), 2.27$ (d, $3 \mathrm{H}, J=1.2 \mathrm{~Hz}, \mathrm{CH}_{3}$ ), 1.45 (h, $\left.2 \mathrm{H}, J=7.6 \mathrm{~Hz}, \mathrm{OCH}_{2} \mathrm{CH}_{2} \mathrm{CH}_{3}\right), 1.38$ (t, $\left.3 \mathrm{H}, J=7.2 \mathrm{~Hz}, \mathrm{CH}_{2} \mathrm{CH}_{3}\right), 0.65$ (t, $3 \mathrm{H}, J=7.2$ $\mathrm{Hz}, \mathrm{O}$ C. $\left.\mathrm{H}_{2} \mathrm{CH}_{2} \mathrm{CH}_{3}\right)$. MS m/z $353\left(\mathrm{M}^{+}+1\right)$; Compound 9: ${ }^{1} \mathrm{H} \mathrm{NMR}\left(400 \mathrm{MHz}, \mathrm{CDCl}_{3}\right.$, ppm): $\delta$ $8.08(\mathrm{~d}, 1 \mathrm{H}, J=8.4 \mathrm{~Hz}, \mathrm{Ar}-H), 7.83(\mathrm{~d}, 1 \mathrm{H}, J=9.2 \mathrm{~Hz}, \mathrm{Ar}-H), 7.55(\mathrm{~d}, 1 \mathrm{H}, J=8.8 \mathrm{~Hz}, \mathrm{Ar}-H), 7.45$ (t, $1 \mathrm{H}, J=8.0 \mathrm{~Hz}, \mathrm{Ar}-H), 7.38(\mathrm{~d}, 1 \mathrm{H}, J=6.8 \mathrm{~Hz}, \mathrm{Ar}-H), 7.33(\mathrm{~d}, 1 \mathrm{H}, J=0.8 \mathrm{~Hz}, \mathrm{OCH}), 3.96(\mathrm{~s}$, $\left.3 \mathrm{H}, \mathrm{OCH}_{3}\right), 3.66\left(\mathrm{~s}, 3 \mathrm{H}, \mathrm{OCH}_{3}\right), 3.11\left(\mathrm{q}, 2 \mathrm{H}, J=6.8 \mathrm{~Hz}, \mathrm{CH}_{2} \mathrm{CH}_{3}\right), 2.28\left(\mathrm{~s}, 3 \mathrm{H}, \mathrm{CH}_{3}\right), 1.39(\mathrm{t}, 3 \mathrm{H}$, $\left.J=6.8 \mathrm{~Hz}, \mathrm{CH}_{2} \mathrm{CH}_{3}\right)$. MS m/z $341\left(\mathrm{M}^{+}+1\right)$; Compound 10: ${ }^{1} \mathrm{H} \mathrm{NMR}\left(400 \mathrm{MHz}, \mathrm{CDCl}_{3}, \mathrm{ppm}\right): \delta$ 8.09 (d, $1 \mathrm{H}, J=8.2 \mathrm{~Hz}, \mathrm{Ar}-H), 7.88(\mathrm{~d}, 1 \mathrm{H}, J=8.8 \mathrm{~Hz}, \mathrm{Ar}-H), 7.50(\mathrm{~m}, 2 \mathrm{H}, \mathrm{Ar}-H), 7.43(\mathrm{t}, 1 \mathrm{H}, J=$ $7.6 \mathrm{~Hz}, \operatorname{Ar}-H), 7.32(\mathrm{~d}, 1 \mathrm{H}, J=1.2 \mathrm{~Hz}, \mathrm{OCH}), 6.39$ (brs, $1 \mathrm{H}, \mathrm{NH}), 3.75\left(\mathrm{~s}, 3 \mathrm{H}, \mathrm{OCH}_{3}\right), 3.10$ (q, $2 \mathrm{H}$, $\left.J=7.6 \mathrm{~Hz}, \mathrm{CH}_{2} \mathrm{CH}_{3}\right), 2.75\left(\mathrm{~d}, 3 \mathrm{H}, J=4.8 \mathrm{~Hz}, \mathrm{NCH}_{3}\right), 2.49\left(\mathrm{~d}, 3 \mathrm{H}, J=1.2 \mathrm{~Hz}, \mathrm{CH}_{3}\right), 1.39(\mathrm{t}, 3 \mathrm{H}, J$ $\left.=7.6 \mathrm{~Hz}, \mathrm{CH}_{2} \mathrm{CH}_{3}\right)$. MS m/z $324\left(\mathrm{M}^{+}+1\right)$; Compound 11: ${ }^{1} \mathrm{H} \mathrm{NMR}\left(400 \mathrm{MHz}, \mathrm{CDCl}_{3}, \mathrm{ppm}\right): \delta$ $8.14(\mathrm{~d}, 1 \mathrm{H}, J=8.4 \mathrm{~Hz}, \mathrm{Ar}-H), 8.00(\mathrm{~d}, 1 \mathrm{H}, J=7.6 \mathrm{~Hz}, \mathrm{Ar}-H), 7.92(\mathrm{~d}, 1 \mathrm{H}, J=8.8 \mathrm{~Hz}, \mathrm{Ar}-H), 7.70$ $(\mathrm{t}, 1 \mathrm{H}, J=7.2 \mathrm{~Hz}, \mathrm{Ar}-H), 7.51(\mathrm{~m}, 3 \mathrm{H}, \mathrm{Ar}-H), 7.37(\mathrm{~m}, 2 \mathrm{H}, \mathrm{Ar}-H \& \mathrm{OCH}), 3.82\left(\mathrm{~s}, 3 \mathrm{H}, \mathrm{OCH}_{3}\right)$, $3.10\left(\mathrm{q}, 2 \mathrm{H}, J=7.6 \mathrm{~Hz}, \mathrm{CH}_{2} \mathrm{CH}_{3}\right), 2.35\left(\mathrm{~d}, 3 \mathrm{H}, J=1.2 \mathrm{~Hz}, \mathrm{CH}_{3}\right), 1.36(\mathrm{t}, 3 \mathrm{H}, J=7.6 \mathrm{~Hz}$, $\left.\mathrm{CH}_{2} \mathrm{CH}_{3}\right)$. MS m/z $428\left(\mathrm{M}^{+}+1\right)$; Compound 12: ${ }^{1} \mathrm{H}$ NMR $\left(300 \mathrm{MHz}, \mathrm{CDCl}_{3}, \mathrm{ppm}\right): \delta 8.08(\mathrm{~d}, 1 \mathrm{H}$, $J=8.4 \mathrm{~Hz}, \operatorname{Ar}-H), 7.83(\mathrm{~d}, 1 \mathrm{H}, J=8.7 \mathrm{~Hz}, \operatorname{Ar}-H), 7.53(\mathrm{~d}, 1 \mathrm{H}, J=9.3 \mathrm{~Hz}, \mathrm{Ar}-H), 7.44(\mathrm{t}, 1 \mathrm{H}, J=$ $8.1 \mathrm{~Hz}, \operatorname{Ar}-H), 7.37(\mathrm{~d}, 1 \mathrm{H}, J=6.3 \mathrm{~Hz}, \operatorname{Ar}-H), 7.32(\mathrm{~d}, 1 \mathrm{H}, J=0.9 \mathrm{~Hz}, \mathrm{OCH}), 3.76(\mathrm{q}, 2 \mathrm{H}, J=7.2$ $\mathrm{Hz}, \mathrm{OCH}_{2} \mathrm{CH}_{3}$ ), 3.67 (s, $3 \mathrm{H}, \mathrm{OCH}_{3}$ ), 3.09 (q, $2 \mathrm{H}, J=7.5 \mathrm{~Hz}, \mathrm{CH}_{2} \mathrm{CH}_{3}$ ), 2.23 (d, $3 \mathrm{H}, J=0.9 \mathrm{~Hz}$, $\left.\mathrm{CH}_{3}\right), 1.38\left(\mathrm{t}, 3 \mathrm{H}, J=7.5 \mathrm{~Hz}, \mathrm{CH}_{2} \mathrm{CH}_{3}\right), 1.26$ (t, $\left.3 \mathrm{H}, J=7.2 \mathrm{~Hz}, \mathrm{OCH}_{2} \mathrm{CH}_{3}\right) . \mathrm{MS} m / z 361$ $\left(\mathrm{M}^{+}+23\right)$; Compound 14: ${ }^{1} \mathrm{H}$ NMR (300 MHz, $\left.\mathrm{CDCl}_{3}, \mathrm{ppm}\right): \delta 8.09(\mathrm{~d}, 1 \mathrm{H}, J=8.4 \mathrm{~Hz}, \mathrm{Ar}-H), 7.90$ $(\mathrm{d}, 1 \mathrm{H}, J=9.0 \mathrm{~Hz}, \operatorname{Ar}-H), 7.55(\mathrm{~d}, 1 \mathrm{H}, J=8.7 \mathrm{~Hz}, \operatorname{Ar}-H), 7.49(\mathrm{dd}, 1 \mathrm{H}, J=7.2,8.1 \mathrm{~Hz}, \mathrm{Ar}-H)$, $7.40(\mathrm{~d}, 1 \mathrm{H}, J=6.3 \mathrm{~Hz}, \mathrm{Ar}-H), 7.37(\mathrm{~d}, 1 \mathrm{H}, J=1.2 \mathrm{~Hz}, \mathrm{OCH}), 4.48\left(\mathrm{~d}, 2 \mathrm{H}, J=6.0 \mathrm{~Hz}, \mathrm{CH}_{2} \mathrm{OH}\right)$, $3.70\left(\mathrm{~s}, 3 \mathrm{H}, \mathrm{OCH}_{3}\right), 3.12\left(\mathrm{q}, 2 \mathrm{H}, J=7.5 \mathrm{~Hz}, \mathrm{CH}_{2} \mathrm{CH}_{3}\right), 2.75(\mathrm{t}, 1 \mathrm{H}, J=6.3 \mathrm{~Hz}, \mathrm{OH}), 2.17(\mathrm{~d}, 3 \mathrm{H}, J$ $\left.=1.2 \mathrm{~Hz}, \mathrm{CH}_{3}\right), 1.39$ (t, $\left.3 \mathrm{H}, J=7.5 \mathrm{~Hz}, \mathrm{CH}_{2} \mathrm{CH}_{3}\right)$. HRMS Calcd for $\mathrm{C}_{19} \mathrm{H}_{21} \mathrm{O}_{3}\left(\mathrm{M}+\mathrm{H}^{+}\right): 297.1485$, found: 297.1470; Compound 15: ${ }^{1} \mathrm{H}$ NMR (400 MHz, $\left.\mathrm{CDCl}_{3}\right): 8.09$ (d, $\left.1 \mathrm{H}, J=8.3 \mathrm{~Hz}, \mathrm{Ar}-H\right), 7.88$ $(\mathrm{d}, 1 \mathrm{H}, J=8.8 \mathrm{~Hz}, \mathrm{Ar}-H), 7.53(\mathrm{~d}, 1 \mathrm{H}, J=8.8 \mathrm{~Hz}, \mathrm{Ar}-H), 7.48(\mathrm{dd}, 1 \mathrm{H}, J=8.4$ and $7.0 \mathrm{~Hz}, \mathrm{Ar}-H)$, 7.40 (d, $1 \mathrm{H}, J=7.0 \mathrm{~Hz}, \mathrm{Ar}-H), 7.38$ (br s, $1 \mathrm{H}, \mathrm{Ar}-H), 4.47$ [s, 2H, $\mathrm{CH}_{2} \mathrm{OH}$ ], 3.85 (q, 2H, $J=7.1 \mathrm{~Hz}$, $\mathrm{OCH}_{2} \mathrm{CH}_{3}$ ), $3.12\left(\mathrm{q}, 2 \mathrm{H}, J=7.5 \mathrm{~Hz}, \mathrm{CH}_{2} \mathrm{CH}_{3}\right), 2.17$ (s, $3 \mathrm{H}, \mathrm{CH}$ ), 1.39 (t, $3 \mathrm{H}, J=7.5 \mathrm{~Hz}$, $\left.\mathrm{CH}_{2} \mathrm{CH}_{3}\right), 1.29\left(\mathrm{t}, 3 \mathrm{H}, J=7.1 \mathrm{~Hz}, \mathrm{OCH}_{2} \mathrm{CH}_{3}\right)$; Compound 16: ${ }^{1} \mathrm{H}$ NMR $\left(400 \mathrm{MHz}, \mathrm{CDCl}_{3}\right): 8.02$ $(\mathrm{d}, 1 \mathrm{H}, J=8.4 \mathrm{~Hz}, \mathrm{Ar}-H), 7.73(\mathrm{~d}, 1 \mathrm{H}, J=8.8 \mathrm{~Hz}, \mathrm{Ar}-H), 7.59(\mathrm{~d}, 1 \mathrm{H}, J=8.8 \mathrm{~Hz}, \mathrm{Ar}-H), 7.47(\mathrm{dd}$, $1 \mathrm{H}, J=8.4$ and $7.0 \mathrm{~Hz}, \mathrm{Ar}-H), 7.39(\mathrm{~d}, 1 \mathrm{H}, J=7.0 \mathrm{~Hz}, \mathrm{Ar}-H), 7.33(\mathrm{br} \mathrm{s}, 1 \mathrm{H}, \mathrm{Ar}-H), 5.05$ [s, 2H, $\left.\mathrm{CH}_{2} \mathrm{OAc}\right], 3.12\left(\mathrm{q}, 2 \mathrm{H}, J=7.5 \mathrm{~Hz}, \mathrm{CH}_{2} \mathrm{CH}_{3}\right), 2.10$ [s, 3H, OC(O) $\left.\mathrm{CH}_{3}\right], 2.03\left(\mathrm{~s}, 3 \mathrm{H}, \mathrm{CH}_{3}\right), 2.36$ [s, $\left.3 \mathrm{H}, \mathrm{OC}(\mathrm{O}) \mathrm{CH}_{3}\right], 2.09\left[\mathrm{~s}, 3 \mathrm{H}, \mathrm{CH}_{2} \mathrm{OC}(\mathrm{O}) \mathrm{CH}_{3}\right], 2.08\left(\mathrm{~s}, 3 \mathrm{H}, \mathrm{CH}_{3}\right), 1.39(\mathrm{t}, 3 \mathrm{H}, J=7.5 \mathrm{~Hz}$,

$\left.\mathrm{CH}_{2} \mathrm{CH}_{3}\right)$. MS m/z $389\left(\mathrm{M}^{+}+\mathrm{Na}\right)$; Compound 17: ${ }^{1} \mathrm{H} \mathrm{NMR}\left(300 \mathrm{MHz}, \mathrm{CDCl}_{3}, \mathrm{ppm}\right): \delta 8.13(\mathrm{~d}, 1 \mathrm{H}$, $J=8.4 \mathrm{~Hz}, \operatorname{Ar}-H), 7.85(\mathrm{~d}, 1 \mathrm{H}, J=9.0 \mathrm{~Hz}, \operatorname{Ar}-H), 7.56(\mathrm{~d}, 1 \mathrm{H}, J=8.7 \mathrm{~Hz}, \operatorname{Ar}-H), 7.47(\mathrm{t}, 1 \mathrm{H}, J=$ $7.2 \mathrm{~Hz}, \mathrm{Ar}-H), 7.40(\mathrm{~d}, 1 \mathrm{H}, J=8.4 \mathrm{~Hz}, \mathrm{Ar}-H), 7.37$ (s, $1 \mathrm{H}, \mathrm{OCH}), 4.40$ (s, $\left.2 \mathrm{H}, \mathrm{CH}_{2} \mathrm{OH}\right), 3.71(\mathrm{~s}, 3 \mathrm{H}$, $\mathrm{OCH}_{3}$ ), 3.32 (s, $3 \mathrm{H}, \mathrm{OCH}_{3}$ ), $3.11\left(\mathrm{q}, 2 \mathrm{H}, J=7.5 \mathrm{~Hz}, \mathrm{CH}_{2} \mathrm{CH}_{3}\right), 2.14$ (s, 3H, CH3), 1.39 (t, $3 \mathrm{H}, J=$ $\left.7.5 \mathrm{~Hz}, \mathrm{CH}_{2} \mathrm{CH}_{3}\right)$. MS m/z $311\left(\mathrm{M}^{+}+1\right)$; Compound 18: ${ }^{1} \mathrm{H} \mathrm{NMR}\left(300 \mathrm{MHz}, \mathrm{CDCl}_{3}, \mathrm{ppm}\right): \delta 8.13$ $(\mathrm{d}, 1 \mathrm{H}, J=8.1 \mathrm{~Hz}, \operatorname{Ar}-H), 7.85(\mathrm{~d}, 1 \mathrm{H}, J=9.0 \mathrm{~Hz}, \operatorname{Ar}-H), 7.57(\mathrm{~d}, 1 \mathrm{H}, J=8.7 \mathrm{~Hz}, \operatorname{Ar}-H), 7.47$ (t, $1 \mathrm{H}, J=8.4 \mathrm{~Hz}, \operatorname{Ar}-H), 7.40(\mathrm{~d}, 1 \mathrm{H}, J=8.4 \mathrm{~Hz}, \operatorname{Ar}-H), 7.35(\mathrm{~d}, 1 \mathrm{H}, J=1.2 \mathrm{~Hz}, \mathrm{OCH}), 4.44(\mathrm{~s}, 2 \mathrm{H}$, $\left.\mathrm{CH}_{2} \mathrm{OH}\right), 3.71\left(\mathrm{~s}, 3 \mathrm{H}, \mathrm{OCH}_{3}\right), 3.48\left(\mathrm{q}, 2 \mathrm{H}, J=7.2 \mathrm{~Hz}, \mathrm{OCH}_{2} \mathrm{CH}_{3}\right), 3.12(\mathrm{q}, 2 \mathrm{H}, J=7.5 \mathrm{~Hz}$, $\mathrm{CH}_{2} \mathrm{CH}_{3}$ ), 2.15 (d, $\left.3 \mathrm{H}, J=1.2 \mathrm{~Hz}, \mathrm{CH}_{3}\right), 1.39$ (t, $\left.3 \mathrm{H}, J=7.5 \mathrm{~Hz}, \mathrm{CH}_{2} \mathrm{CH}_{3}\right), 1.20$ (t, $3 \mathrm{H}, J=7.2 \mathrm{~Hz}$, $\left.\mathrm{OCH}_{2} \mathrm{CH}_{3}\right)$. MS m/z $325\left(\mathrm{M}^{+}+1\right)$; Compound 19: ${ }^{1} \mathrm{H} \mathrm{NMR}\left(400 \mathrm{MHz}, \mathrm{CDCl}_{3}\right): 8.09(\mathrm{~d}, 1 \mathrm{H}, J=$ $8.4 \mathrm{~Hz}, \operatorname{Ar}-H), 7.88(\mathrm{~d}, 1 \mathrm{H}, J=8.8 \mathrm{~Hz}, \operatorname{Ar}-H), 7.55(\mathrm{~d}, 1 \mathrm{H}, J=8.8 \mathrm{~Hz}, \operatorname{Ar}-H), 7.49(\mathrm{dd}, 1 \mathrm{H}, J=8.4$ and 7.2 Hz, Ar- $H$ ), 7.44-7.35 (m, 2H, Ar- $H$ ), 4.49 [s, $\left.2 \mathrm{H}, \mathrm{CH}_{2} \mathrm{Br}\right], 3.70$ (s, 3H, OCH $), 3.11$ (q, $2 \mathrm{H}$, $\left.J=7.4 \mathrm{~Hz}, \mathrm{CH}_{2} \mathrm{CH}_{3}\right), 2.17\left(\mathrm{~s}, 3 \mathrm{H}, \mathrm{CH}_{3}\right), 1.39\left(\mathrm{t}, 3 \mathrm{H}, J=7.4 \mathrm{~Hz}, \mathrm{CH}_{2} \mathrm{CH}_{3}\right)$; Compound 20: ${ }^{1} \mathrm{H}$ $\operatorname{NMR}\left(400 \mathrm{MHz}, \mathrm{CDCl}_{3}\right): 8.12(\mathrm{~d}, 1 \mathrm{H}, J=8.4 \mathrm{~Hz}, \mathrm{Ar}-H), 7.86(\mathrm{~d}, 1 \mathrm{H}, J=8.8 \mathrm{~Hz}, \mathrm{Ar}-H), 7.55(\mathrm{~d}$, $1 \mathrm{H}, J=8.8 \mathrm{~Hz}, \mathrm{Ar}-H), 7.47(\mathrm{dd}, 1 \mathrm{H}, J=8.4$ and $7.2 \mathrm{~Hz}, \mathrm{Ar}-H), 7.41-7.38(\mathrm{~m}, 2 \mathrm{H}, \mathrm{Ar}-H), 4.92$ [s, $\left.2 \mathrm{H}, \mathrm{CH}_{2} \mathrm{OCH}_{2} \mathrm{CF}_{3}\right], 3.71\left(\mathrm{~s}, 3 \mathrm{H}, \mathrm{OCH}_{3}\right), 3.49\left(\mathrm{t}, 2 \mathrm{H}, J=9.4 \mathrm{~Hz}, \mathrm{OCH}_{2} \mathrm{CF}_{3}\right), 3.11(\mathrm{q}, 2 \mathrm{H}, J=7.4$ $\left.\mathrm{Hz}, \mathrm{CH}_{2} \mathrm{CH}_{3}\right), 2.17$ (s, 3H, $\left.\mathrm{CH}_{3}\right), 1.39\left(\mathrm{t}, 3 \mathrm{H}, \mathrm{J}=7.4 \mathrm{~Hz}, \mathrm{CH}_{2} \mathrm{CH}_{3}\right) . \mathrm{MS} \mathrm{m} / z, 402\left(\mathrm{M}^{+}+\mathrm{Na}\right)$; 
Compound 21: ${ }^{1} \mathrm{H}$ NMR (400 MHz, $\left.\mathrm{CDCl}_{3}\right): 8.11(\mathrm{~d}, 1 \mathrm{H}, J=8.4 \mathrm{~Hz}, \mathrm{Ar}-H), 7.86(\mathrm{~d}, 1 \mathrm{H}, J=8.8$ $\mathrm{Hz}, \mathrm{Ar}-H), 7.54(\mathrm{~d}, 1 \mathrm{H}, J=8.8 \mathrm{~Hz}, \mathrm{Ar}-H), 7.46(\mathrm{dd}, 1 \mathrm{H}, J=8.4$ and 7.2 Hz, Ar- $H), 7.40-7.36(\mathrm{~m}$, $2 \mathrm{H}, \mathrm{Ar}-\mathrm{H}), 5.10$ [s, $\left.2 \mathrm{H}, \mathrm{CH}_{2} \mathrm{OC}(\mathrm{O})-\right], 3.70\left(\mathrm{~s}, 3 \mathrm{H}, \mathrm{OCH}_{3}\right), 3.11\left(\mathrm{q}, 2 \mathrm{H}, J=7.4 \mathrm{~Hz}, \mathrm{CH}_{2} \mathrm{CH}_{3}\right), 2.11$ [s, 3H, C(O)CH $\left.\mathrm{CH}_{3}\right), 2.04\left(\mathrm{~s}, 3 \mathrm{H}, \mathrm{CH}_{3}\right) 1.39$ (t, 3H, J = 7.4 Hz, $\left.\mathrm{CH}_{2} \mathrm{CH}_{3}\right) . \mathrm{MS} \mathrm{m} / z 339\left(\mathrm{M}_{-}^{+} 1\right)$; Compound 22: ${ }^{1} \mathrm{H}$ NMR (400 MHz, $\left.\mathrm{CDCl}_{3}\right): 8.10(\mathrm{~d}, 1 \mathrm{H}, J=8.4 \mathrm{~Hz}, \mathrm{Ar}-H), 7.84(\mathrm{~d}, 1 \mathrm{H}, J=8.8$ $\mathrm{Hz}, \mathrm{Ar}-H), 7.54$ (d, $1 \mathrm{H}, J=8.8 \mathrm{~Hz}, \mathrm{Ar}-H), 7.46(\mathrm{dd}, 1 \mathrm{H}, J=8.4$ and $7.2 \mathrm{~Hz}, \mathrm{Ar}-H), 7.40-7.36$ (m, $2 \mathrm{H}, \mathrm{Ar}-\mathrm{H}), 5.11$ [s, $\left.2 \mathrm{H}, \mathrm{CH}_{2} \mathrm{OC}(\mathrm{O})-\right], 3.70\left(\mathrm{~s}, 3 \mathrm{H}, \mathrm{OCH}_{3}\right), 3.10$ (q, $\left.2 \mathrm{H}, J=7.5 \mathrm{~Hz}, \mathrm{CH}_{2} \mathrm{CH}_{3}\right), 2.74$ (t, $\left.2 \mathrm{H}, J=7.5 \mathrm{~Hz}, \mathrm{CH}_{2} \mathrm{CH}_{2} \mathrm{NEt}_{2}\right), 2.45\left[\mathrm{q}, 4 \mathrm{H}, J=7.2 \mathrm{~Hz}, \mathrm{~N}\left(\mathrm{CH}_{2} \mathrm{CH}_{3}\right)_{2}\right.$ ], 2.42 [t, $2 \mathrm{H}, J=7.2 \mathrm{~Hz}$, $\mathrm{C}(\mathrm{O})-\mathrm{CH}_{2} \mathrm{CH}_{2} \mathrm{NEt}_{2}$ ], $2.11\left(\mathrm{~s}, 3 \mathrm{H}, \mathrm{CH}_{3}\right) 1.39\left(3 \mathrm{H}, \mathrm{t}, J=7.5 \mathrm{~Hz}, \mathrm{CH}_{2} \mathrm{CH}_{3}\right), 0.97$ [t, $6 \mathrm{H}, J=7.2 \mathrm{~Hz}$, $\left.\mathrm{N}\left(\mathrm{CH}_{2} \mathrm{CH}_{3}\right)_{2}\right]$. MS m/z $424\left(\mathrm{M}^{+}-1\right)$; Compound 23: ${ }^{1} \mathrm{H}$ NMR $\left(400 \mathrm{MHz}, \mathrm{CDCl}_{3}\right): 8.13(\mathrm{~d}, 1 \mathrm{H}, J=$ $8.4 \mathrm{~Hz}, \operatorname{Ar}-H), 7.84(\mathrm{~d}, 1 \mathrm{H}, J=8.8 \mathrm{~Hz}, \mathrm{Ar}-H), 7.55(\mathrm{~d}, 1 \mathrm{H}, J=8.8 \mathrm{~Hz}, \mathrm{Ar}-H), 7.45(\mathrm{dd}, 1 \mathrm{H}, J=8.4$ and $7.0 \mathrm{~Hz}, \mathrm{Ar}-H), 7.40-7.33(\mathrm{~m}, 2 \mathrm{H}, \mathrm{Ar}-H), 4.40$ [s, $2 \mathrm{H}, \mathrm{CH}_{2} \mathrm{OMe}$, $3.82(\mathrm{q}, 2 \mathrm{H}, J=7.0 \mathrm{~Hz}$, $\mathrm{OCH}_{2} \mathrm{CH}_{3}$ ), $3.30\left(\mathrm{~s}, 3 \mathrm{H}, \mathrm{CH}_{2} \mathrm{OCH}_{3}\right), 3.11$ (q, $\left.2 \mathrm{H}, \mathrm{J}=7.5 \mathrm{~Hz}, \mathrm{CH}_{2} \mathrm{CH}_{3}\right), 2.14$ (s, 3H, $\left.\mathrm{CH}_{3}\right), 1.39$ (t, $\left.3 \mathrm{H}, J=7.5 \mathrm{~Hz}, \mathrm{CH}_{2} \mathrm{CH}_{3}\right), 1.30\left(\mathrm{t}, 3 \mathrm{H}, J=7.0 \mathrm{~Hz}, \mathrm{OCH}_{2} \mathrm{CH}_{3}\right)$; Compound 24: ${ }^{1} \mathrm{H}$ NMR (400 $\left.\mathrm{MHz}_{\mathrm{CDCl}}\right): 8.13(\mathrm{~d}, 1 \mathrm{H}, J=8.4 \mathrm{~Hz}, \mathrm{Ar}-H), 7.84(\mathrm{~d}, 1 \mathrm{H}, J=8.8 \mathrm{~Hz}, \mathrm{Ar}-H), 7.55(\mathrm{~d}, 1 \mathrm{H}, J=8.8$ $\mathrm{Hz}, \operatorname{Ar}-H), 7.44(\mathrm{dd}, 1 \mathrm{H}, J=8.4$ and $7.0 \mathrm{~Hz}, \mathrm{Ar}-H), 7.40-7.35(\mathrm{~m}, 2 \mathrm{H}, \mathrm{Ar}-H), 5.10[\mathrm{~s}, 2 \mathrm{H}$, $\mathrm{CH}_{2} \mathrm{OAc}$ ], $3.82\left(\mathrm{q}, 2 \mathrm{H}, J=7.0 \mathrm{~Hz}, \mathrm{OCH}_{2} \mathrm{CH}_{3}\right), 3.10\left(\mathrm{q}, 2 \mathrm{H}, J=7.5 \mathrm{~Hz}, \mathrm{CH}_{2} \mathrm{CH}_{3}\right), 2.10$ [s, $3 \mathrm{H}$, $\left.\mathrm{OC}(\mathrm{O}) \mathrm{CH}_{3}\right], 2.03\left(\mathrm{~s}, 3 \mathrm{H}, \mathrm{CH}_{3}\right), 1.39\left(\mathrm{t}, 3 \mathrm{H}, J=7.5 \mathrm{~Hz}, \mathrm{CH}_{2} \mathrm{CH}_{3}\right), 1.30(\mathrm{t}, 3 \mathrm{H}, J=7.0 \mathrm{~Hz}$, $\left.\mathrm{OCH}_{2} \mathrm{CH}_{3}\right)$. MS $m / z, 353\left(\mathrm{M}^{+}-1\right)$.

7. Miki Y, Swensen J, Shattuck-Eidens D, Futreal PA, Harshman K, Tavtigian S, Liu Q, Cochran C, Bennett LM, Ding W, Bell R, Rosentha J, Hussey C, Tran T, McClure M, Frye C, Hattier T, Phelps R, Haugne-Strano A, Katcher H, Yakumo K, Gholami Z, Shaffer D, Stone S, Bayer S, Wray C, Bogden R, Dayananth P, Ward J, Tonin P, Narod S, Bristow PK, Norris FH, Helvering L, Morrison P, Rosteck P, Lai M, Barrett JC, Lewis C, Neuhausen S, Cannon-Albright L, Goldgar D, Wiseman R, Kamb A, Skolnick JH. Science 1994;266:66. [PubMed: 7545954]

8. Moynahan ME. Oncogene 2002;21:8994. [PubMed: 12483515]

9. Turner N, Tutt A, Ashworth A. Nat. Rev. Cancer 2004;4:814. [PubMed: 15510162]

10. Ting NS, Lee WH. DNA Repair 2004;3:935. [PubMed: 15279779]

11. Poole AJ, Li Y, Kim Y, Lin S-CJ, Lee W-H, Lee EYHP. Science 2006;314:1467. [PubMed: 17138902]

12. Brcal ${ }^{f p / f p_{p 53} f p / f p_{C r e} \text { Mutant Mice. Generation of } B r c a l}{ }^{f p / f p_{p 5} f p / f p_{C r e}}$ and $p 53^{f p / f p}$ Cre mice has been described previously. ${ }^{11,13}$. The mice were in a C57BL/6 and 129/Sv, mixed background. All animal experiments were in accordance with guidelines of federal and Institutional Animal Care and Use Committee at the University of California, Irvine. Treatment with 3. Three-monthold mice were treated with $0.1 \mathrm{mg}$ of $\mathbf{3}$ or vehicle daily for 11 days. Stock solution of $\mathbf{3}$ was 10 $\mathrm{mg} / \mathrm{mL}$ in dimethylsulfoxide. A mixture of $10 \mu \mathrm{L}$ of stock solution, $30 \mu \mathrm{L}$ of $40 \%$ polyethylene glycol, and $60 \mu \mathrm{L}$ of $0.9 \% \mathrm{NaCl}$ solution was prepared at the time of treatment. Vehicle includes all solution except 3. Vehicle or compound was administered i.p. every day for 11 days.

13. Shafee N, Smith CR, Wei S, Kim Y, Mills GB, Hortobagyi GN, Stanbridge EJ, Lee EY. Cancer Res 2008;68:3243. [PubMed: 18451150]

14. Histology and Immunohistochemistry. The fourth pair glands were dissected and spread on a glass slide. After fixation with Carnoy's fixative for three $h$, the tissues were hydrated and stained in Carmine alum overnight as described

(http://mammary.nih.gov/tools/histological/Histology/index.html\#a1). Branching points in three random areas totaling approximately $2 \mathrm{~mm}^{2}$ were counted. For histological section, tissues were fixed in $4 \%$ paraformaldehyde (Sigma-Aldrich, St. Louis, MO) at $4{ }^{\circ} \mathrm{C}$ overnight followed by paraffin embedding. Paraffin sections were stained with hematoxylin and eosin and examined by light microscopy. Immunostaining was performed following the protocol described in the Vectastain Elite ABC kit (Vector Laboratories, Burlingame, CA.) To retrieve the antigen, slides were heated for $20 \mathrm{~min}$ in $10 \mathrm{mM}$ citrate buffer, $\mathrm{pH}$ 6.0, in a microwave oven. BrdU monoclonal antibody (GeneTex Inc. Irvine, CA) at 1:1000 dilution, and cyclin D1 polyclonal rat antibody (NeoMarkers/Thermo Fisher Scientific, Fremont, CA) at 1:500 dilution, respectively, were used for immunostaining.

15. LaMarca HL, Rosen JM. Endocrinology 2008;149:4317. [PubMed: 18556345]

16. Lee EY-HP. Curr. Opin. Obstet. Gynecol 2008;20:68. [PubMed: 18197009] 
17. Lim E, Vaillant F, Wu D, Forrest NC, Pal B, Hart AH, Asselin-Labat ML, Gyorki DE, Ward T, Partanen A, Feleppa F, Huschtscha LI, Thorne HJ, kConFab; Fox SB, Yan M, French JD, Brown MA, Smyth GK, Visvader JE, Lindeman GJ. Nat. Med 2009;15:907. [PubMed: 19648928] 

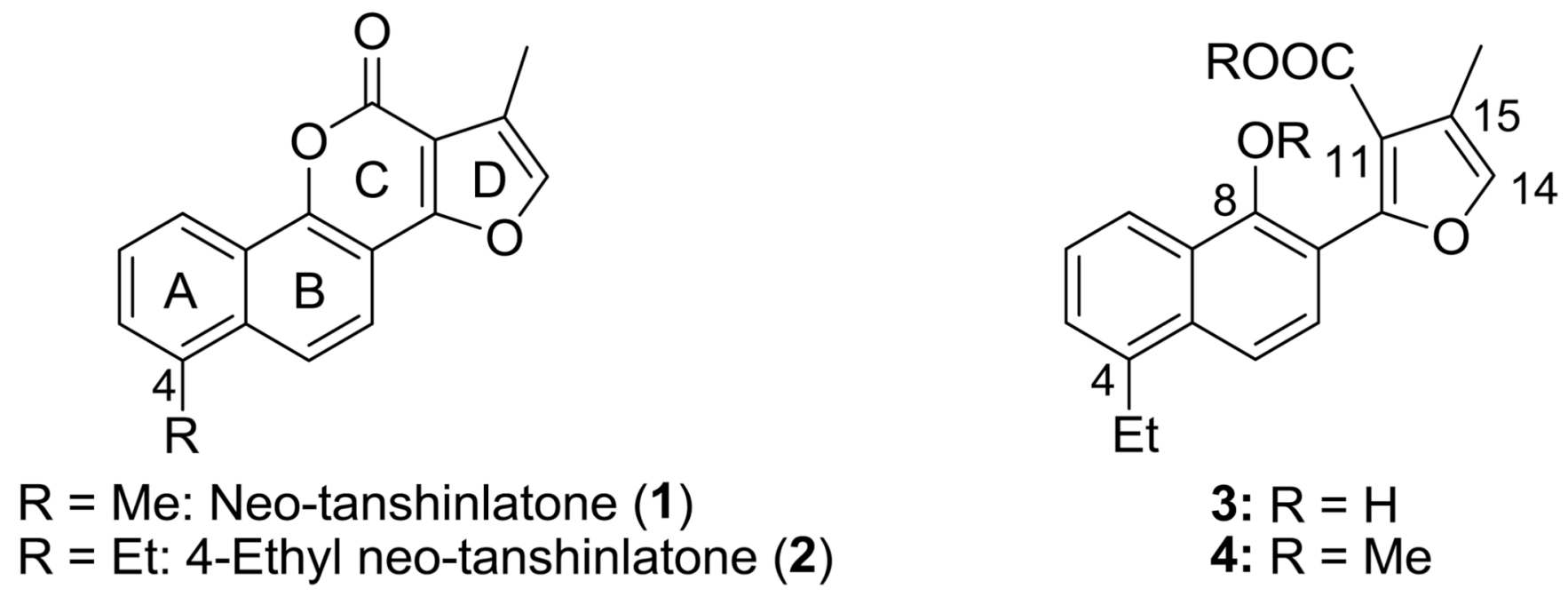

Figure 1.

Structures of neo-tanshinlactone (1), 4-ethyl neo-tanshinlactone (2), and FNO analogs $\mathbf{3}$ and 4 


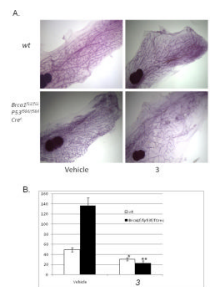

Figure 2.

Treatment with $\mathbf{3}$ leads to decreased mammary ductal branching. Mammary gland whole mounts were prepared from wild-type and Brcal ${ }^{f 11 / f 11} p 53^{f 5 \& 6 / f 5 \& 6} \mathrm{Cre}^{c}$ mice following treatment with $0.1 \mathrm{mg}$ of $\mathbf{3}$ daily for 11 days. (A) Mammary gland whole mounts of vehicle(a \& c) and 3-treated (b \& d) 3-month-old mice. (B) Number of branching points in wildtype (wt) and Brcal ${ }^{f 11 / f 11}$ p5 $3^{f 5 \& 6 / f 5 \& 6} \mathrm{Cre} e^{c}$ mammary glands. The data represent average of branch points in five randomly selected areas $\pm \mathrm{SD} .(* \mathrm{P} \leq 0.002 ; * * \mathrm{P} \leq 0.0002)$ 


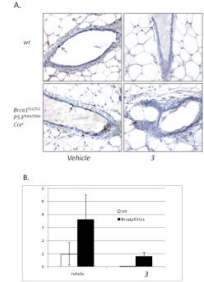

Figure 3.

Treatment with 3 leads to decreased mammary epithelial proliferation. BrdU-containing drinking water was provided during the last three days of $\mathbf{3}$ treatment. (A) Cells uptaking BrdU, indicative of DNA synthesis, were detected by immunostaining. Arrows indicate BrdU-positive cells. (B) Quantification of BrdU-positive cells in 15 mammary ducts. Average number of BrdU-positive cells in vehicle- and 3-treated wild-type ( $w t)$ and Brcal ${ }^{f 11 / f 11} p 53^{f 5 \& 6 / f 5 \& 6} C r e^{c}$ mice. Arrows indicate alveoli. Histogram shows the average number of BrdU labeled cells per duct $\pm \mathrm{SD}(* \mathrm{P} \leq 0.002 ; * * \mathrm{P} \leq 0.0001)$. At least 15 mammary ducts/animal were evaluated (a minimum of three mice per genotype). Mammary glands from vector $(\mathrm{a} \& \mathrm{c})$ or $\mathbf{3}$ (b \& d) treated mice were removed and fixed with paraformaldehyde. Paraffin sections were labeled with antibody to cyclin D1. At least 15 mammary ducts/animal were evaluated. 


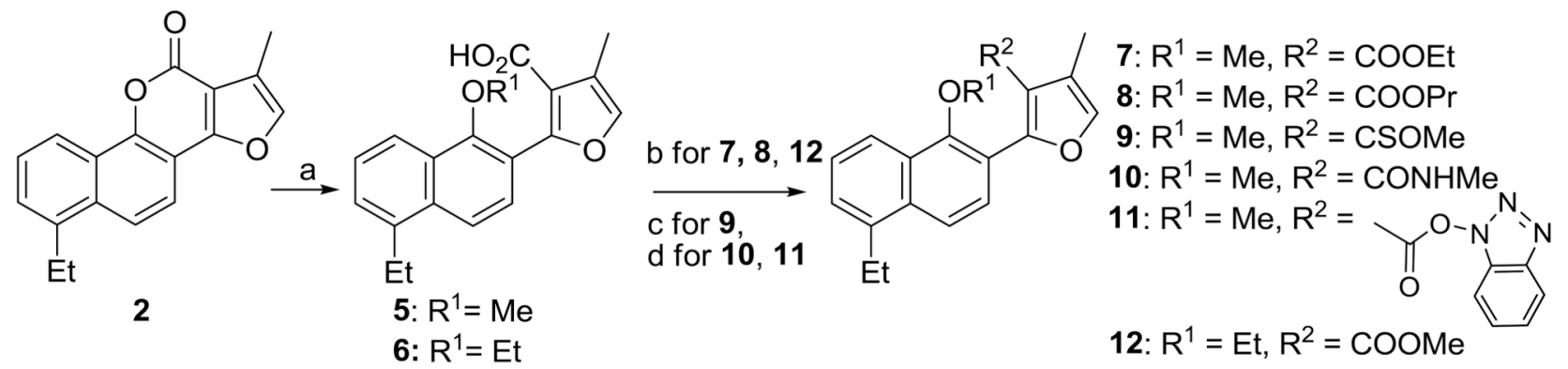

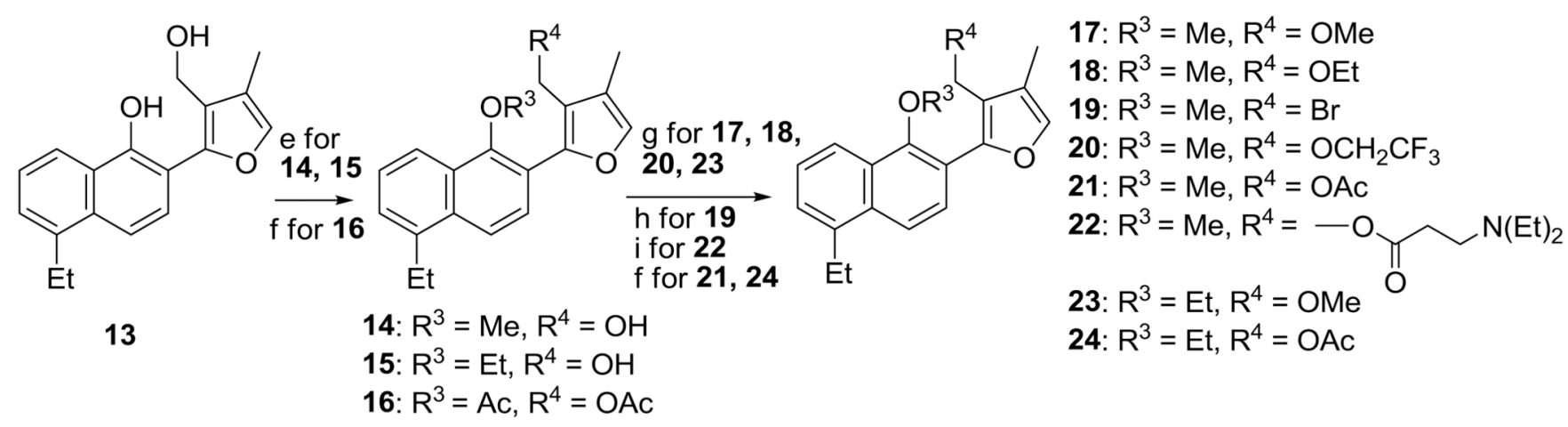

Scheme 1.

Reactions and conditions: (a) $\mathrm{NaOH}, 18$-crown-6, $\mathrm{R}_{1} \mathrm{I}, \mathrm{CH}_{3} \mathrm{CN}, 90{ }^{\circ} \mathrm{C}$; (b) $\mathrm{SOCl}_{2}, \mathrm{R}_{2} \mathrm{OH}$, rt; (c) (i) $\mathrm{SOCl}_{2}, \mathrm{MeOH}$, rt; (ii) Lawesson's reagent, toluene, reflux, 5h; (d) HOBt, EDCI, $\mathrm{CH}_{2} \mathrm{Cl}_{2},\left(\mathrm{H}_{2} \mathrm{NMe}\right.$ for 10); (e) MeI or EtI, $\mathrm{Cs}_{2} \mathrm{CO}_{3}$, acetone, rt; (f) $\mathrm{Ac} 2 \mathrm{O}, \mathrm{Py}, \mathrm{DMAP}, 90{ }^{\circ} \mathrm{C}$; (g) MeI or EtI, $\mathrm{NaH}, \mathrm{THF}, \mathrm{rt},\left(\mathrm{CF}_{3} \mathrm{CH}_{2} \mathrm{I}, \mathrm{DMF}, 0{ }^{\circ} \mathrm{C}\right.$ for 20); (h) $\mathrm{PPh}_{3}, \mathrm{Br}_{2}$, imidazole, $0{ }^{\circ} \mathrm{C}$ to rt; (i) EDCI, DMAP, 3-(diethylamino)-propanoic acid hydrochloride, $\mathrm{CH}_{2} \mathrm{Cl}_{2}$, rt. 
